\title{
Predicting agronomic performance of barley using canopy reflectance data
}

\author{
T. G. Fetch, Jr. ${ }^{1}$, B. J. Steffenson ${ }^{2}$, and V. D. Pederson ${ }^{3, \dagger}$ \\ ${ }^{1}$ Cereal Research Centre, Agriculture and Agri-Food Canada, Winnipeg, Manitoba, Canada R3T 2M9 \\ (e-mail address: tfetch@agr.gc.ca); ${ }^{2}$ Department of Plant Pathology, University of Minnesota, St Paul, \\ MN, USA 55108-6030; and ${ }^{3}$ Department of Plant Pathology, North Dakota State University, Fargo, ND, USA \\ 58105. Published as paper number 1849 in the contribution series from the Cereal Research Centre of \\ Agriculture and Agri-Food Canada. Received 23 December 2001, accepted 30 June 2003.
}

Fetch, T. G., Jr., Steffenson, B. J. and Pederson, V. D. 2004. Predicting agronomic performance of barley using canopy reflectance data. Can. J. Plant Sci. 84: 1-9. The ability to accurately and rapidly predetermine agronomic performance would be desirable in most plant breeding programs. Remote sensing of canopy reflectance is a quick and nondestructive method that may be useful in the estimation of agronomic performance. Studies were conducted at Fargo and Langdon, North Dakota, to determine the effectiveness of a multispectral radiometer in estimating yield, kernel plumpness (KP), and 1000-kernel weight (TKW) in barley. Canopy reflectance was measured in eight $(500-850 \mathrm{~nm})$ discrete narrow-wavelength bands. Three types of reflectance models were evaluated: simple models using one to four wavelengths, simple ratio and normalized difference vegetation indices (NDVI) using green, red, and near-infrared wavelengths, and soil-adjusted vegetation indices (SAVI). The relationship between canopy reflectance and agronomic performance was significantly influenced by environment, growth stage, and plant genotype. Grain yield was best estimated near GS73 $\left(0.84<R^{2}<0.92\right)$ at Fargo and at GS83 $\left(0.55<R^{2}<0.81\right)$ at Langdon. In contrast, KP and TKW could be estimated at both late (GS83; $0.68<R^{2}<0.93$ ) and early (GS24-GS47; $0.72<R^{2}<0.91$ ) growth stages. The 550-nm and 800-nm wavelengths are critical for development of predictive models. A simple model using 550-nm, 600-nm, and 800-nm from GS47-GS73 gave significant $\left(0.45<R^{2}<0.64\right)$ estimation of agronomic performance across all environments. In contrast, simple ratio, NDVI, and SAVI were less effective $\left(0.05<R^{2}<0.77\right)$ in predicting agronomic performance. Remote sensing using canopy reflectance is a potential tool to estimate agronomic performance of barley, but genotypic and crop stage factors affect this method. Further studies are needed to improve the usefulness of multispectral radiometry in predicting agronomic performance.

Key words: Crop yield, Hordeum vulgare, kernel plumpness, remote sensing

Fetch, T. G., Jr., Steffenson, B. J. et Pederson, V. D. 2004. Prévision du rendement de l'orge d'après la réflexion de la couverture végétale. Can. J. Plant Sci. 84: 1-9. Pouvoir déterminer rapidement à l'avance et avec précision le rendement bénéficierait à la plupart de programmes d'hybridation. Mesurer la réflexion de la couverture végétale par télédétection est une technique rapide et non destructive qui pourrait avoir son utilité à cet égard. Les auteurs ont entrepris des études à Fargo et à Langdon, au Dakota Nord, pour vérifier dans quelle mesure un radiomètre multispectral permettrait d'estimer le rendement, la rondeur du grain (RG) et le poids de 1000 grains (PMG) de l'orge. Ils ont mesuré la réflexion de la couverture végétale à huit petites longueurs d'onde ponctuelles (de 500 à $850 \mathrm{~nm}$ ) puis évalué trois sortes de modèles reposant sur la réflexion : un modèle simple utilisant de une à quatre longueurs d'onde, un second reposant sur un rapport simple et un indice normalisé de variation de la végétation (INVV) reposant sur la longueur d'onde du vert, du rouge et du proche infrarouge, et un troisième reposant sur un indice de la végétation corrigé pour le sol (IVCS). Le lien entre la réflexion et le rendement subit sensiblement l'influence du milieu, du stade de croissance (SC) et du génotype de la plante. Les auteurs ont obtenu la meilleure estimation du rendement grainier au SC73 $\left(0,84<R^{2}<0,92\right)$, à Fargo, et au SC83 $\left(0,55<R^{2}<0,81\right)$, à Langdon. La RG et le PMG sont plus faciles à estimer vers la fin (SC83; 0,68 $<R^{2}<0,93$ ) et au début (SC24-SC47; $0,72<R^{2}<0,91$ ) de la croissance. Les longueurs d'onde de 550 et $800 \mathrm{~nm}$ sont indispensables à l'élaboration de modèles de prévision. Un modèle simple reposant sur les longueurs d'onde de 550 , 600 et $800 \mathrm{~nm}$ du SC47 au SC73 permet une estimation sensible $\left(0,45<\mathrm{R}^{2}<0,64\right)$ du rendement dans tous les milieux. En revanche, le modèle à rapport simple et INVV et le modèle IVCS sont moins efficaces $\left(0,05<R^{2}<0,77\right)$. La réflexion de la couverture végétale mesurée par télédétection pourrait devenir un instrument utile pour estimer le rendement de l'orge, mais le génotype et le stade de croissance affectent cette technique. Il faudrait entreprendre d'autres recherches afin d'accroître l'utilité de la radiométrie multispectrale pour prévoir le rendement.

Mots clés: Rendement, Hordeum vulgare, rondeur du grain, télédétection

$\dagger$ Deceased.
Abbreviations: KP, kernel plumpness; LAI, leaf area index; MCARI, modified chlorophyll absorption reflectance index; NDVI, normalized difference vegetation indices; NIR, nearinfrared reflectance; OSAVI, optimum soil-adjusted vegetation index; SAVI, soil-adjusted vegetation indices; TKW, thousand-kernel weight; VIs, vegetative indices 


\begin{tabular}{|c|c|c|c|c|c|c|c|c|c|c|}
\hline \multirow[b]{2}{*}{ Genotype $^{\mathbf{z}}$} & \multicolumn{5}{|c|}{ Fargo } & \multicolumn{5}{|c|}{ Langdon } \\
\hline & 1987 & 1988 & 1989 & 1990 & 1991 & 1987 & 1988 & 1989 & 1990 & 1991 \\
\hline & \multicolumn{10}{|c|}{ Yield $\left(\right.$ g plot $\left.^{-1}\right)$} \\
\hline Azure & $1279 a$ & $119 a$ & $1523 a$ & $3058 a$ & $1415 a$ & $1705 a$ & $1493 a$ & $2195 a$ & $2698 a$ & $2082 a$ \\
\hline Larker & $1147 b$ & $102 a$ & $1572 a$ & $2588 b$ & $1109 b$ & $1743 a$ & $1427 a$ & $2227 a$ & $2334 b$ & $1689 b$ \\
\hline \multirow[t]{2}{*}{ ND B112 } & - & $110 a$ & $1516 a$ & $2037 c$ & $1149 b$ & - & $1142 b$ & $1719 b$ & $1859 c$ & $1411 c$ \\
\hline & \multicolumn{10}{|c|}{ Kernel plumpness (\%) } \\
\hline Azure & $72.2 a$ & $47.9 a$ & $81.5 a$ & $82.5 a$ & $51.2 a$ & $72.2 b$ & $83.1 a$ & $83.2 a$ & $95.9 a$ & $66.4 a$ \\
\hline Larker & $71.6 a$ & $47.5 a$ & $74.0 b$ & $76.5 b$ & $48.5 b$ & $77.0 a$ & $84.2 a$ & $76.9 b$ & $92.1 b$ & $63.5 a$ \\
\hline \multirow[t]{2}{*}{ ND B112 } & - & $32.6 b$ & $59.0 c$ & $71.6 c$ & $41.8 c$ & - & $59.9 b$ & $63.3 c$ & $87.4 c$ & $58.4 b$ \\
\hline & \multicolumn{10}{|c|}{ 500-kernel weight $\left(\mathrm{g} \mathrm{plot}^{-1}\right)$} \\
\hline Azure & $18.3 a$ & $-\mathbf{y}$ & $19.0 a$ & $19.3 a$ & $15.1 a$ & $18.3 b$ & $19.4 b$ & $19.7 a$ & $21.1 a$ & $17.2 a$ \\
\hline Larker & $17.9 b$ & - & $18.3 b$ & $19.0 a$ & $15.1 a$ & $18.7 a$ & $20.4 a$ & $18.7 b$ & $19.9 c$ & $16.6 b$ \\
\hline ND B112 & - & - & $17.3 c$ & $19.2 a$ & $15.1 a$ & - & $18.1 c$ & $17.7 c$ & $20.3 b$ & $16.6 b$ \\
\hline
\end{tabular}

${ }^{{ }^{2}}$ ND B112 was not planted in 1987.

y Due to extremely low plot yields in 1988, there was not enough seed to perform 500-kernel weights.

$a-c$ Means followed by the same letter are not significantly different $(P>0.05)$ using Duncan's Multiple Range Test.

Crop improvement, which usually can be measured by parameters such as higher yield and/or improved quality and agronomic traits, is the primary goal of most plant breeders. These crop improvement parameters are often influenced by environmental, pest, nutritional, or other factors. Accurate estimation of agronomic performance, particularly at growth stages prior to harvest, could be extremely useful to plant breeders for making decisions as to inclusion of lines or populations for further advancement and testing. Additionally, early estimation of potential yield may be valuable to producers or crop consultants for decisions concerning additional crop inputs. A promising tool that has potential for early and accurate estimates of agronomic performance, particularly over large geographical areas, is remote sensing.

Remote sensing of crops by measurement of canopy reflectance at specific wavelengths has been used previously to estimate crop health and potential yield (Bauer 1975; Knipling 1970). Most yield models have been derived using various kinds of crop growth modeling that is dependent on the prediction of leaf area index (LAI), amount of absorbed photosynthetically active radiation, or fraction of absorbed photosynthetically active radiation over the growing season (Clevers 1997). Of these variables, LAI appears to be the most important for use in yield prediction models. Various equations have been proposed to estimate LAI based on leaf reflectance values in the red $(600-700 \mathrm{~nm})$ and nearinfrared (800-1100 nm) ranges (Clevers 1988; Maas 1993). However, prediction of LAI is often complicated by factors such as crop growth stage, where reflectance data are influenced by the relative proportion of canopy cover to soil background (Lorenzen and Jensen 1991), and also by cultivar effects (Ahlrichs and Bauer 1983).

There also has been considerable use of vegetation indices (VIs) based on simple ratios or combinations of visible and near infrared reflectance (NIR) wavelengths for estimation of parameters such as LAI, green biomass and yield (Aase et al. 1986; Ma et al. 2001; Maas 1993; Wiegand and Richardson 1990; Wiegand et al. 1979, 1991).
Vegetation indices are useful for estimation of biomass and LAI because they attempt to minimize the influences of changing light intensity and soil reflectivity by use of differences between plants and soil backgrounds in their reflectance in the red and NIR wavelengths (Hatfield and Pintar 1993). However, sensitivity of VIs to atmospheric and soil background effects led to development of soiladjusted indices such as the optimum soil-adjusted vegetation index (OSAVI) (Rondeaux et al. 1996) and modified chlorophyll absorption reflectance index (MCARI) (Daughtry et al. 2000).

The primary objective of this research was to develop simple predictive models using crop canopy reflectance values to estimate yield, KP, and TKW in barley. Since the crop stage of development and cultivar type have previously been determined to influence canopy reflectance, these variables were also studied for their effects on the predictive models. Additionally, VIs were examined for their usefulness in predicting the agronomic parameters listed above.

\section{MATERIALS AND METHODS}

Barley (Hordeum vulgare L.) plots were grown at Fargo (Fargo silty clay) and Langdon (Svea loam), North Dakota from 1987 to 1991. The experimental design was a $3 \times 9$ factorial that included three barley genotypes (Azure, Larker, ND B112) and nine treatments [untreated check and combinations of seed (Imazalil at $13 \mathrm{~mL} \mathrm{~kg}^{-1}$ or $39 \mathrm{~mL} \mathrm{~kg}^{-1}$ ) and foliar (Tilt at $287 \mathrm{~mL} \mathrm{ha}^{-1}$ or Mancozeb at $2.24 \mathrm{~kg} \mathrm{ha}^{-1}$ ) fungicide applications] in a randomized complete block with four replicates. Barley genotypes and fungicide applications were selected to obtain maximum differences in agronomic performance and in susceptibility to foliar plant diseases. Seed was planted $\left(45 \mathrm{~g} \mathrm{plot}^{-1}\right)$ each year into land that was fallow the previous growing season and fertilized in the fall for a target yield of about $4 \mathrm{tha}^{-1}$. Plot size was $1.5 \mathrm{~m} \times 3.5 \mathrm{~m}$ (6 rows/plot with ca. 20 -cm row spacing) with $1.5-\mathrm{m}$ winter wheat alleys surrounding each plot. Herbicides were applied at GS15 (Zadoks et al. 1974) for postemergence weed control. Hand weeding was also done to remove 

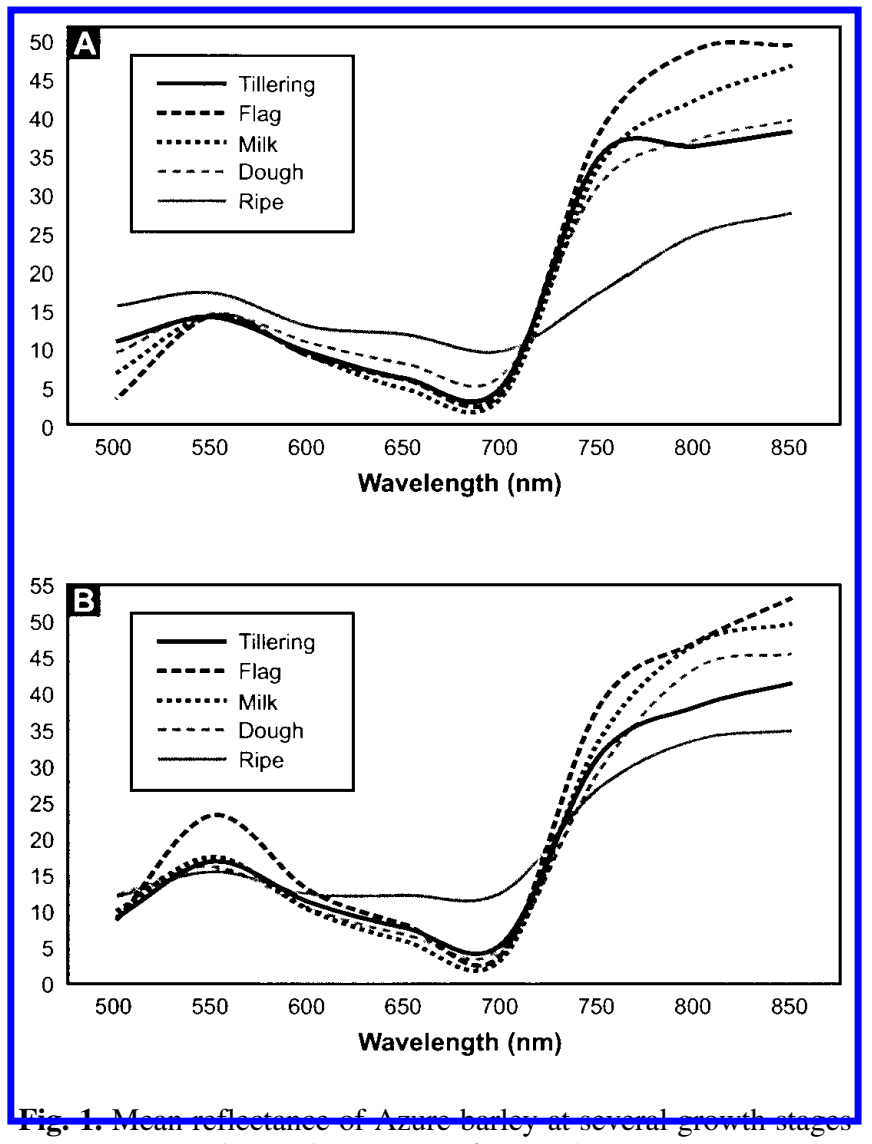

at Fargo (A) and Langdon (B), ND from 1987 to 1991.

all unwanted plants from the test plots. Plots were inoculated with leaf-spotting fungi (Bipolaris sorokiniana in Fargo and Drechslera teres f. teres in Langdon) using conidial sprays (GS51, 1987-1989) or disease-infested straw (GS24, 1990-1991) (Fetch and Steffenson 1994; Steffenson et al. 1996) to induce leaf infection and to increase variability in agronomic performance. Designated plots were sprayed with foliar fungicides when the barley heads were just emerging (GS51).

\section{REFLECTANCE MEASUREMENTS}

Canopy reflectance was measured using a multispectral radiometer (MSR87, CropScan ${ }^{\circledR}$ Inc., Rochester, MN). This radiometer records canopy reflectance at eight wavelengths $(500-850 \mathrm{~nm}$ at $50-\mathrm{nm}$ intervals) with a $10-\mathrm{nm}$ bandwidth and a $28^{\circ}$ field of vision (Pederson and Fiechtner 1980). The CropScan ${ }^{\circledR}$ radiometer records both incoming and reflected solar radiation simultaneously, thus accounting for effects of cloud cover on canopy reflectance. Percent reflectance of the canopy for each wavelength was determined by an algorithm where: Percent reflectance $=$ (incident calibrated constant/reflected calibrated constant $) \times($ reflected irradiation/ Incident radiation) $\times 100 \%$. Percent canopy reflectance for each wavelength is calculated in real-time using the constant values, incident radiation, and reflected radiation, and stored in a portable computer. Two readings were taken at opposite ends of each plot and average percent canopy reflectance for each wavelength was recorded. The portable computer also recorded Julian day and time, environmental conditions, growth stage, plot number, incident solar radiation, and temperature at the time of measurement. The radiometer sensors were mounted on a hand-held aluminum boom and elevated $2 \mathrm{~m}$ above the crop canopy. At this height, the sensor assessed a circular area $1 \mathrm{~m}$ in diameter within the plot. The radiometer was calibrated at the beginning of each sampling date using a diffusing opal glass alternatively held over the incident and reflective sensors facing the same incident sunlight. This calibrated the incident and reflective sensors relative to each other. Radiometer readings of the plots were obtained within $2 \mathrm{hr}$ of solar noon on relatively cloud-free and windless days. About $30 \mathrm{~s}$ per plot was required for acquisition and recording of radiometric data. Canopy reflection of plots was obtained at the tillering (GS24), flag leaf sheath opening (GS47), early milk (GS73), early dough (GS83), and kernel ripe (GS91) stages of development at each location.

\section{Agronomic Measurements}

Plant emergence was determined at the two-leaf stage (GS12) by counting the number of seedlings/meter in two randomly chosen rows within each plot. Plots were harvested at maturity with a small plot combine except in 1988, where plots were hand-harvested due to extremely low yields caused by drought. Harvested seeds were dried at $30^{\circ} \mathrm{C}$ for $2 \mathrm{~d}$ in a grain dryer to a moisture level of 12-13\% (Model TY9304 grain moisture tester, John Deere ${ }^{\circledR}$ Inc., Moline, IL), then weighed to determine total plot yields. Kernel plumpness was determined on two random 100-g subsamples from each plot according to American Society of Brewing Chemists (ASBC) method Barley-2C (ASBC 1992) using a Niagara sample grader (Niagara Inc., Niagara, NY). The subsample was poured onto the top sieve, the machine turned on for $30 \mathrm{~s}$, and the percentage of plump kernels remaining on the top sieve (2.38- $\mathrm{mm}$ by $1.91-\mathrm{mm}$ slots) was calculated by weight. Very dry conditions in 1988 resulted in most plots with yields of less than 1000 kernels; therefore, TKW was calculated by measuring the weight of 500 randomly selected barley seeds from all plots for all years.

\section{Statistical Analysis}

Data were analyzed using SAS Institute, Inc. procedures (SAS Institute, Inc. 1999). Analysis of variance (ANOVA) procedures evaluated the relationships between independent (barley genotype and fungicide treatments) and dependent (plant emergence, yield, KP and TKW) variables. $F$-values and Probability $>F$ statistics were calculated, and the level of significance was pre-established at $\alpha=0.05$. Bartlett's test for homogeneity of variance also was calculated to determine if data could be pooled across locations and years.

The relationship between the canopy reflectance data and the agronomic variables was evaluated using regression analysis (PROC REG). In order to determine the type of regression model(s) that would be most useful, plots of the reflectance data and individual agronomic variables were generated (PROC PLOT). Several regression models were tested using 


\begin{tabular}{|c|c|c|c|c|c|c|c|c|c|}
\hline \multirow{2}{*}{$\begin{array}{l}\text { Barley } \\
\text { genotype }\end{array}$} & \multirow[b]{2}{*}{ GS $^{y}$} & \multicolumn{2}{|c|}{ Best one wavelength $\mathbf{z}$} & \multicolumn{2}{|c|}{ Best two wavelengths $\mathbf{z}$} & \multicolumn{2}{|c|}{ Best three wavelengths ${ }^{\mathbf{z}}$} & \multicolumn{2}{|c|}{ Best four wavelengths ${ }^{\mathbf{z}}$} \\
\hline & & Band & $R^{2}$ & Bands & $R^{2}$ & Bands & $R^{2}$ & Bands & $R^{2}$ \\
\hline Azure & 24 & 500 & 0.127 & 500,600 & 0.198 & $500,550,850$ & 0.205 & $500,600,800,850$ & 0.214 \\
\hline Larker & 24 & 850 & 0.217 & 500,600 & 0.482 & $500,550,650$ & 0.511 & $500,550,650,800$ & 0.518 \\
\hline ND B112 & 24 & 750 & 0.481 & 600,650 & 0.519 & $550,600,650$ & 0.542 & $550,600,650,750$ & 0.558 \\
\hline Combined & 24 & 850 & 0.174 & 500,600 & 0.295 & $500,650,800$ & 0.331 & $500,650,800,850$ & 0.341 \\
\hline Azure & 47 & 550 & 0.899 & 600,700 & 0.904 & $500,550,850$ & 0.909 & $550,650,750,850$ & 0.910 \\
\hline Larker & 47 & 550 & 0.855 & 700,750 & 0.871 & $750,800,850$ & 0.876 & $550,750,800,850$ & 0.880 \\
\hline ND B112 & 47 & 800 & 0.426 & 700,800 & 0.471 & $550,650,800$ & 0.523 & $550,650,800,850$ & 0.530 \\
\hline Combined & 47 & 550 & 0.765 & 500,700 & 0.801 & $500,600,700$ & 0.814 & $500,550,600,700$ & 0.822 \\
\hline Azure & 73 & 700 & 0.731 & 550,600 & 0.796 & $550,800,850$ & 0.847 & $650,700,800,850$ & 0.912 \\
\hline Larker & 73 & 800 & 0.755 & 550,800 & 0.812 & $550,800,850$ & 0.899 & $500,550,800,850$ & 0.920 \\
\hline ND B112 & 73 & 800 & 0.894 & 800,850 & 0.917 & $550,800,850$ & 0.926 & $650,700,800,850$ & 0.942 \\
\hline Combined & 73 & 800 & 0.726 & 750,800 & 0.795 & $550,800,850$ & 0.869 & $650,700,800,850$ & 0.902 \\
\hline Azure & 83 & 550 & 0.312 & 500,600 & 0.727 & $500,600,650$ & 0.755 & $500,550,650,700$ & 0.803 \\
\hline Larker & 83 & 550 & 0.238 & 500,600 & 0.707 & $500,600,800$ & 0.752 & $700,750,800,850$ & 0.785 \\
\hline ND B112 & 83 & 800 & 0.559 & 800,850 & 0.712 & $750,800,850$ & 0.740 & $650,700,750,800$ & 0.746 \\
\hline Combined & 83 & 550 & 0.143 & 500,600 & 0.580 & $500,600,800$ & 0.615 & $500,550,650,700$ & 0.693 \\
\hline Azure & 91 & 550 & 0.195 & 500,550 & 0.217 & $500,550,700$ & 0.277 & $500,550,700,850$ & 0.301 \\
\hline Larker & 91 & 500 & 0.235 & 500,700 & 0.344 & $500,650,800$ & 0.353 & $500,600,650,750$ & 0.360 \\
\hline ND B112 & 91 & 550 & 0.359 & 800,850 & 0.401 & $500,550,650$ & 0.448 & $500,550,650,700$ & 0.456 \\
\hline Combined & 91 & 550 & 0.091 & 550,600 & 0.130 & $500,550,600$ & 0.140 & $500,550,800,850$ & 0.158 \\
\hline
\end{tabular}

${ }^{\mathrm{z} B e s t}$ wavelength models were generated using the MAX $R^{2}$ option in PROC REG using SAS Institute, Inc. Software (1999).

$\mathbf{y}_{\mathrm{GS}}=$ growth stage of plants when radiometer data was collected, as defined using Zadoks (1974) scale, where GS24 = tillering, GS47 = flag leaf, GS73 = early milk, GS83 = early dough, and GS91 = kernel ripe.

\begin{tabular}{|c|c|c|c|c|c|c|c|c|c|}
\hline \multirow{2}{*}{$\begin{array}{l}\text { Barley } \\
\text { genotype }\end{array}$} & \multirow[b]{2}{*}{ GS $^{\mathbf{y}}$} & \multicolumn{2}{|c|}{ Best one wavelength ${ }^{\mathbf{z}}$} & \multicolumn{2}{|c|}{ Best two wavelengths $\mathbf{z}$} & \multicolumn{2}{|c|}{ Best three wavelengths $\mathbf{z}$} & \multicolumn{2}{|c|}{ Best four wavelengths ${ }^{\mathbf{Z}}$} \\
\hline & & Band & $R^{2}$ & Bands & $R^{2}$ & Bands & $R^{2}$ & Bands & $R^{2}$ \\
\hline Azure & 24 & 700 & 0.459 & 650,750 & 0.589 & $700,800,850$ & 0.622 & $500,750,800,850$ & 0.668 \\
\hline Larker & 24 & 850 & 0.058 & 700,850 & 0.438 & $550,650,750$ & 0.511 & $500,750,800,850$ & 0.563 \\
\hline ND B112 & 24 & 550 & 0.189 & 550,700 & 0.309 & $600,650,700$ & 0.321 & $500,550,650,700$ & 0.333 \\
\hline Combined & 24 & 700 & 0.054 & 550,700 & 0.265 & $700,800,850$ & 0.347 & $500,750,800,850$ & 0.374 \\
\hline Azure & 47 & 850 & 0.625 & 750,800 & 0.672 & $600,750,800$ & 0.694 & $500,600,750,800$ & 0.707 \\
\hline Larker & 47 & 800 & 0.487 & 500,800 & 0.511 & $550,600,700$ & 0.598 & $550,600,650,750$ & 0.602 \\
\hline ND B112 & 47 & 800 & 0.226 & 500,550 & 0.339 & $500,550,850$ & 0.352 & $500,550,650,800$ & 0.366 \\
\hline Combined & 47 & 800 & 0.194 & 550,600 & 0.340 & $600,750,800$ & 0.374 & $550,600,750,800$ & 0.376 \\
\hline Azure & 73 & 700 & 0.498 & 550,800 & 0.638 & $700,750,800$ & 0.674 & $500,550,600,800$ & 0.693 \\
\hline Larker & 73 & 700 & 0.237 & 750,800 & 0.488 & $550,800,850$ & 0.543 & $550,700,800,850$ & 0.570 \\
\hline ND B112 & 73 & 600 & 0.125 & 550,600 & 0.257 & $600,650,700$ & 0.300 & $600,650,700,850$ & 0.304 \\
\hline Combined & 73 & 700 & 0.171 & 750,800 & 0.206 & $650,750,800$ & 0.293 & $550,700,800,850$ & 0.315 \\
\hline Azure & 83 & 850 & 0.417 & 550,850 & 0.795 & $500,550,850$ & 0.814 & $500,600,700,850$ & 0.820 \\
\hline Larker & 83 & 850 & 0.725 & 650,850 & 0.766 & $500,550,850$ & 0.794 & $500,550,750,850$ & 0.801 \\
\hline ND B112 & 83 & 800 & 0.525 & 800,850 & 0.548 & $650,800,850$ & 0.551 & $500,550,650,700$ & 0.558 \\
\hline Combined & 83 & 850 & 0.541 & 650,800 & 0.626 & $500,550,850$ & 0.658 & $500,550,750,800$ & 0.663 \\
\hline Azure & 91 & 550 & 0.446 & 500,600 & 0.595 & $500,550,850$ & 0.677 & $500,550,650,850$ & 0.684 \\
\hline Larker & 91 & 550 & 0.266 & 500,700 & 0.552 & $500,550,800$ & 0.592 & $500,550,700,800$ & 0.595 \\
\hline ND B112 & 91 & 800 & 0.140 & 800,850 & 0.314 & $550,800,850$ & 0.318 & $550,650,700,750$ & 0.351 \\
\hline Combined & 91 & 550 & 0.082 & 500,700 & 0.280 & $500,550,750$ & 0.348 & $500,550,650,750$ & 0.354 \\
\hline
\end{tabular}

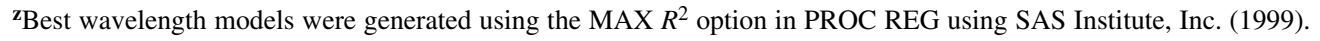

$\mathbf{y}_{\mathrm{GS}}=$ growth stage of plants when radiometer data was collected, as defined using Zadoks $(1974)$ scale, where GS24 = tillering, GS47 = flag leaf, GS73 = early milk, GS83 = early dough, and GS91 = kernel ripe.

the radiometer data: (1) 1, 2, 3, and 4-variable models using narrow band reflectance wavelengths; (2) simple ratio and NDVI indices; and (3) soil-adjusted vegetation indices (SAVI). Models from regression procedures were evaluated using: (1) the $F$-statistic in the ANOVA that tests the overall significance of the model; and (2) the coefficient of multiple determination $\left(R^{2}\right)$ value that indicates the proportion of total variation of the dependent variable that is explained by the overall model. 


\begin{tabular}{|c|c|c|c|c|c|c|c|}
\hline \multirow{2}{*}{$\begin{array}{l}\text { Barley } \\
\text { genotype }\end{array}$} & \multirow[b]{2}{*}{$\mathrm{GS}^{\mathbf{y}}$} & \multirow{2}{*}{$\frac{\mathrm{NIR} / \mathrm{R}^{\mathbf{z}}}{R^{2}}$} & \multirow{2}{*}{$\frac{\text { NDVI } \operatorname{Red}^{\mathbf{z}}}{R^{2}}$} & \multirow{2}{*}{$\frac{\mathrm{NIR} / \mathrm{G}^{\mathbf{Z}}}{R^{2}}$} & \multirow{2}{*}{$\frac{\text { NDVI Green }^{\mathbf{z}}}{R^{2}}$} & \multirow{2}{*}{$\frac{\text { MCARI }^{\mathbf{z}}}{R^{2}}$} & \multirow{2}{*}{$\frac{\text { OSAVI }^{\mathbf{z}}}{R^{2}}$} \\
\hline & & & & & & & \\
\hline Azure & 24 & 0.020 & 0.010 & 0.153 & 0.178 & 0.099 & 0.163 \\
\hline Larker & 24 & 0.100 & 0.194 & 0.028 & 0.032 & 0.001 & 0.031 \\
\hline ND B112 & 24 & 0.381 & 0.378 & 0.245 & 0.227 & 0.495 & 0.038 \\
\hline Combined & 24 & 0.042 & 0.126 & 0.030 & 0.029 & 0.001 & 0.031 \\
\hline Azure & 47 & 0.497 & 0.189 & 0.527 & 0.734 & 0.728 & 0.409 \\
\hline Larker & 47 & 0.484 & 0.226 & 0.584 & 0.764 & 0.743 & 0.531 \\
\hline ND B112 & 47 & 0.239 & 0.256 & 0.187 & 0.200 & 0.005 & 0.250 \\
\hline Combined & 47 & 0.520 & 0.258 & 0.567 & 0.712 & 0.701 & 0.506 \\
\hline Azure & 73 & 0.752 & 0.759 & 0.327 & 0.346 & 0.049 & 0.570 \\
\hline Larker & 73 & 0.752 & 0.764 & 0.377 & 0.429 & 0.003 & 0.649 \\
\hline ND B112 & 73 & 0.653 & 0.727 & 0.269 & 0.273 & 0.166 & 0.667 \\
\hline Combined & 73 & 0.674 & 0.739 & 0.280 & 0.322 & 0.019 & 0.592 \\
\hline Azure & 83 & 0.083 & 0.008 & 0.173 & 0.168 & 0.162 & 0.080 \\
\hline Larker & 83 & 0.028 & 0.002 & 0.120 & 0.109 & 0.051 & 0.055 \\
\hline ND B112 & 83 & 0.001 & 0.022 & 0.237 & 0.271 & 0.109 & 0.248 \\
\hline Combined & 83 & 0.037 & 0.001 & 0.073 & 0.065 & 0.026 & 0.024 \\
\hline Azure & 91 & 0.017 & 0.017 & 0.050 & 0.034 & 0.007 & 0.002 \\
\hline Larker & 91 & 0.169 & 0.179 & 0.014 & 0.018 & 0.164 & 0.150 \\
\hline ND B112 & 91 & 0.052 & 0.055 & 0.001 & 0.001 & 0.018 & 0.074 \\
\hline Combined & 91 & 0.006 & 0.013 & 0.024 & 0.019 & 0.001 & 0.011 \\
\hline
\end{tabular}

${ }^{\mathrm{z}_{\mathrm{NIR}} / \mathrm{R}}=$ near infrared/red reflectance ratio using reflectance at 850 and $700 \mathrm{~nm}$; NDVI Red $=$ normalized difference vegetation index (Red) using the following wavelengths and formula: $\left(\mathrm{R}_{850}-\mathrm{R}_{700}\right) /\left(\mathrm{R}_{850}+\mathrm{R}_{700}\right) ; \mathrm{NIR} / \mathrm{G}=$ near infrared/green reflectance ratio using reflectance at $850 \mathrm{and} 550 \mathrm{~nm} ; \mathrm{NDVI}$ Green $=$ normalized difference vegetation index (Green) using the following wavelengths and formula: $\left(\mathrm{R}_{850}-\mathrm{R}_{550}\right) /\left(\mathrm{R}_{850}+\mathrm{R}_{550}\right)$; MCARI $=$ modified chlorophyll absorption in reflectance index using the reflectance wavelengths and formula: $\left[\left(R_{700}-R_{650}\right)-0.2\left(R_{700}-R_{550}\right)\right] \times\left(R_{700} / R_{650}\right)$; and OSAVI = optimized soil adjusted vegetation index using the reflectance wavelengths and formula: $\left(R_{800}-R_{650}\right) /\left(R_{800}+R_{650}+0.16\right)$.

$\mathbf{y}_{\mathrm{GS}}=$ growth stage of plants when radiometer data was collected, as defined using Zadoks (1974) scale, where GS24 = tillering, GS47 = flag leaf, GS73 = early milk, GS83 = early dough, and GS91 = kernel ripe.

\section{RESULTS AND DISCUSSION}

\section{Agronomic Performance}

Since soil background effects are known to influence spectral reflectance of a plant canopy (Huete 1988; Rondeaux et al. 1996), we seeded at moderately high rates $\left(45 \mathrm{~g} \mathrm{plot}^{-1}=1.6\right.$ bu $\mathrm{A}^{-1}$ ) to minimize soil reflectance contributions and measured the stand establishment for each plot. Stand establishment was uniform (mean of about 200 plants $\mathrm{m}^{-2}$ ) within test plots. Significant differences in stand counts were found among cultivars across environments (data not shown), but were not related to the significant differences found in agronomic performance (Table 1). Plots at Langdon did exhibit about a $7 \%$ higher mean stand compared to plots at Fargo within the same season. Fungicide treatments had no significant effect on stand establishment or agronomic performance (data not presented). This is unexpected, but can be explained by the dry environmental conditions, which are not favorable for disease development.

Bartlett's homogeneity test detected highly significant differences in agronomic performance across environments due to high variability (severe drought in 1988, dry conditions in 1989, ideal growing conditions in 1990, normal in 1987 and 1991) over the 5 yr of field tests. Statistical analyses were performed separately at each environment and for combined environments. When the environments were combined, data were weighted to normalize the variance (Steel and Torrie 1980). Weighted regression models were con- structed using the WEIGHT option and the inverse of the variance term for each dependent variable (yield, KP, TKW) at each environment (location and year). Error variance terms between the environments were rendered homogenous by this procedure. ANOVA also found significant differences in agronomic performance among the genotypes (Table 1). The cultivars Azure and Larker generally had significantly higher yield, KP, and TKW than the genotype ND B112. Since significant differences were detected among the genotypes for all agronomic parameters tested, statistical analyses to evaluate the usefulness of canopy reflectance data in predicting agronomic performance were conducted both for each genotype and also for combined genotypes at each environment.

\section{Narrow Band Reflectance Regression Models}

Since values for canopy reflectance were significantly different at specific wavelengths among the stages of crop development (Fig. 1), regression analyses to develop predictive models were conducted across growth stages and also for each growth stage. Data were pooled across years at each location and regression statistics were calculated for each genotype at various stages of development. Relationships between reflectance data using narrow-band wavelengths and yield for each growth stage and genotype at the two locations are presented in Tables 2 and 3. One-, two-, three-, and four-variable models with the highest $R^{2}$ 


\begin{tabular}{|c|c|c|c|c|c|c|c|}
\hline \multirow{2}{*}{$\begin{array}{l}\text { Barley } \\
\text { genotype }\end{array}$} & \multirow[b]{2}{*}{$\mathrm{GS}^{\mathbf{y}}$} & \multirow{2}{*}{$\frac{\mathrm{NIR} / \mathrm{R}^{\mathbf{z}}}{R^{2}}$} & \multirow{2}{*}{$\frac{\text { NDVI } \operatorname{Red}^{\mathbf{z}}}{R^{2}}$} & \multirow{2}{*}{$\frac{\mathrm{NIR} / \mathrm{G}^{\mathbf{Z}}}{R^{2}}$} & \multirow{2}{*}{$\frac{\text { NDVI Green }^{\mathbf{z}}}{R^{2}}$} & \multirow{2}{*}{$\frac{\mathrm{MCARI}^{\mathbf{z}}}{R^{2}}$} & \multirow{2}{*}{$\frac{\mathrm{OSAVI}^{\mathbf{z}}}{R^{2}}$} \\
\hline & & & & & & & \\
\hline Azure & 24 & 0.147 & 0.369 & 0.133 & 0.142 & 0.045 & 0.238 \\
\hline Larker & 24 & 0.029 & 0.018 & 0.101 & 0.077 & 0.006 & 0.044 \\
\hline ND B112 & 24 & 0.164 & 0.098 & 0.141 & 0.155 & 0.042 & 0.001 \\
\hline Combined & 24 & 0.002 & 0.041 & 0.011 & 0.008 & 0.018 & 0.058 \\
\hline Azure & 47 & 0.649 & 0.608 & 0.158 & 0.196 & 0.051 & 0.052 \\
\hline Larker & 47 & 0.356 & 0.358 & 0.028 & 0.034 & 0.001 & 0.098 \\
\hline ND B112 & 47 & 0.005 & 0.001 & 0.076 & 0.071 & 0.081 & 0.117 \\
\hline Combined & 47 & 0.237 & 0.172 & 0.029 & 0.039 & 0.009 & 0.013 \\
\hline Azure & 73 & 0.512 & 0.549 & 0.456 & 0.393 & 0.036 & 0.122 \\
\hline Larker & 73 & 0.242 & 0.296 & 0.174 & 0.083 & 0.001 & 0.001 \\
\hline ND B112 & 73 & 0.085 & 0.088 & 0.072 & 0.076 & 0.070 & 0.103 \\
\hline Combined & 73 & 0.107 & 0.116 & 0.084 & 0.065 & 0.010 & 0.010 \\
\hline Azure & 83 & 0.001 & 0.014 & 0.106 & 0.214 & 0.571 & 0.085 \\
\hline Larker & 83 & 0.103 & 0.200 & 0.030 & 0.104 & 0.559 & 0.001 \\
\hline ND B112 & 83 & 0.362 & 0.356 & 0.231 & 0.227 & 0.387 & 0.001 \\
\hline Combined & 83 & 0.145 & 0.216 & 0.001 & 0.037 & 0.396 & 0.013 \\
\hline Azure & 91 & 0.388 & 0.344 & 0.466 & 0.461 & 0.001 & 0.401 \\
\hline Larker & 91 & 0.285 & 0.240 & 0.240 & 0.126 & 0.001 & 0.216 \\
\hline ND B112 & 91 & 0.029 & 0.031 & 0.125 & 0.136 & 0.053 & 0.120 \\
\hline Combined & 91 & 0.075 & 0.055 & 0.089 & 0.061 & 0.009 & 0.058 \\
\hline
\end{tabular}

${ }^{\mathbf{z}} \mathrm{NIR} / \mathrm{R}=$ near infrared/red reflectance ratio using reflectance at 850 and $700 \mathrm{~nm}$; NDVI Red = normalized difference vegetation index (Red) using the following wavelengths and formula: $\left(\mathrm{R}_{850}-\mathrm{R}_{700}\right) /\left(\mathrm{R}_{850}+\mathrm{R}_{700}\right) ; \mathrm{NIR} / \mathrm{G}=$ near infrared/green reflectance ratio using reflectance at 850 and 550 nm; NDVI Green $=$ normalized difference vegetation index (Green) using the following wavelengths and formula: $\left(\mathrm{R}_{850}-\mathrm{R}_{550}\right) /\left(\mathrm{R}_{850}+\mathrm{R}_{550}\right)$; MCARI $=$ modified chlorophyll absorption in reflectance index using the reflectance wavelengths and formula: $\left[\left(\mathrm{R}_{700}-\mathrm{R}_{650}\right)-0.2\left(\mathrm{R}_{700}-\mathrm{R}_{550}\right)\right] \times\left(\mathrm{R}_{700} / \mathrm{R}_{650}\right)$; and OSAVI $=$ optimized soil adjusted vegetation index using the reflectance wavelengths and formula: $\left(R_{800}-R_{650}\right) /\left(R_{800}+R_{650}+0.16\right)$.

$\mathbf{y}_{\mathrm{GS}}=$ growth stage of plants when radiometer data was collected, as defined using Zadoks $(1974)$ scale, where GS24 = tillering, GS47 = flag leaf, GS73 = early milk, GS83 = early dough, and GS91 = kernel ripe.

values are presented. Significant improvements in the regression model were found with two-variable models compared to one-variable models and usually with threevariable models compared to two-variable models $(P>F$ statistic in Type III SS). Use of two- or three-variable models was found to be best for prediction of agronomic performance, which agrees with a similar study conducted by Thenkabail et al. (2000).

Crop growth stage had strong effects on the robustness ( $R^{2}$ value) of predictive models, which agrees with previous studies (Ahlrichs and Bauer 1983; Wiegand et al. 1991). Canopy reflectance at the ripe (GS91) stage was poor for estimation of agronomic performance. Yield was best predicted (highest $R^{2}$ value) at the flag leaf to milk stage (GS47-GS73) in Fargo, but at the early dough stage (GS83) in Langdon. The reason for this is unclear, but plots at Langdon did have slightly higher (7\%) stands and generally yielded much higher than at the Fargo location. Additionally, the Langdon environment is cooler than Fargo and promotes longer periods of vegetative and grain filling; thus, the yield potential is determined later at the Langdon site compared to the Fargo site. It is possible that under higher productivity conditions, yield may better be predicted at later growth stages. Plant biomass and LAI are near maximum in the barley canopy at about GS73 (Lorenzen and Jensen 1991). Since LAI has previously been found to be important in developing predictive yield models (Maas 1993; Clevers 1997), it is logical that yield would be best estimated at a growth stage where LAI is near maximum. The wavelengths that were most useful for yield estimation were the 500-, 550-, 800-, and 850-nm bands. The 550-nm (green) band is where reflectance in the visible region is at the peak and has previously been determined to have strong associations with crop variables (Thenkabail et al. 2000). The 800- and 850-nm bands are in the NIR region, which has been found to be strongly associated with LAI (Ahlrichs and Bauer 1983). Our results also agree with a recent yield prediction model developed by Clevers (1997), where sugar beet yield was predicted $\left(R^{2}=0.98\right)$ with a model that used reflectance in the green and NIR bands to estimate the fraction of absorbed photosyntheticaly active radiation at a single growth stage during the season.

In contrast to yield, KP and TKW could be predicted at either early (GS24-GS47) or late (GS83) growth stages and with different narrow band reflectance wavelengths (data not shown). At the Fargo location, KP and TKW had the highest $R^{2}$ values at the tillering stage (GS24) for the cultivars Azure and Larker, but at the dough stage (GS83) for ND B112. The reason for this is unclear, but ND B112 is slightly later in maturity and also has about $20 \%$ more LAI (Fetch, unpublished data), which may account for the cultivar differences in the predictive models. The 500-, 650-, and 700 -nm wavelengths were used to estimate $\mathrm{KP}\left(R^{2}=0.72\right.$ to $0.83)$ and TKW $\left(R^{2}=0.79\right)$ for Azure and Larker, while the 500-, 800-, and 850-nm wavelengths were used to estimate $\mathrm{KP}\left(R^{2}=0.89\right)$ and TKW $\left(R^{2}=0.91\right)$ for ND B112. At the 

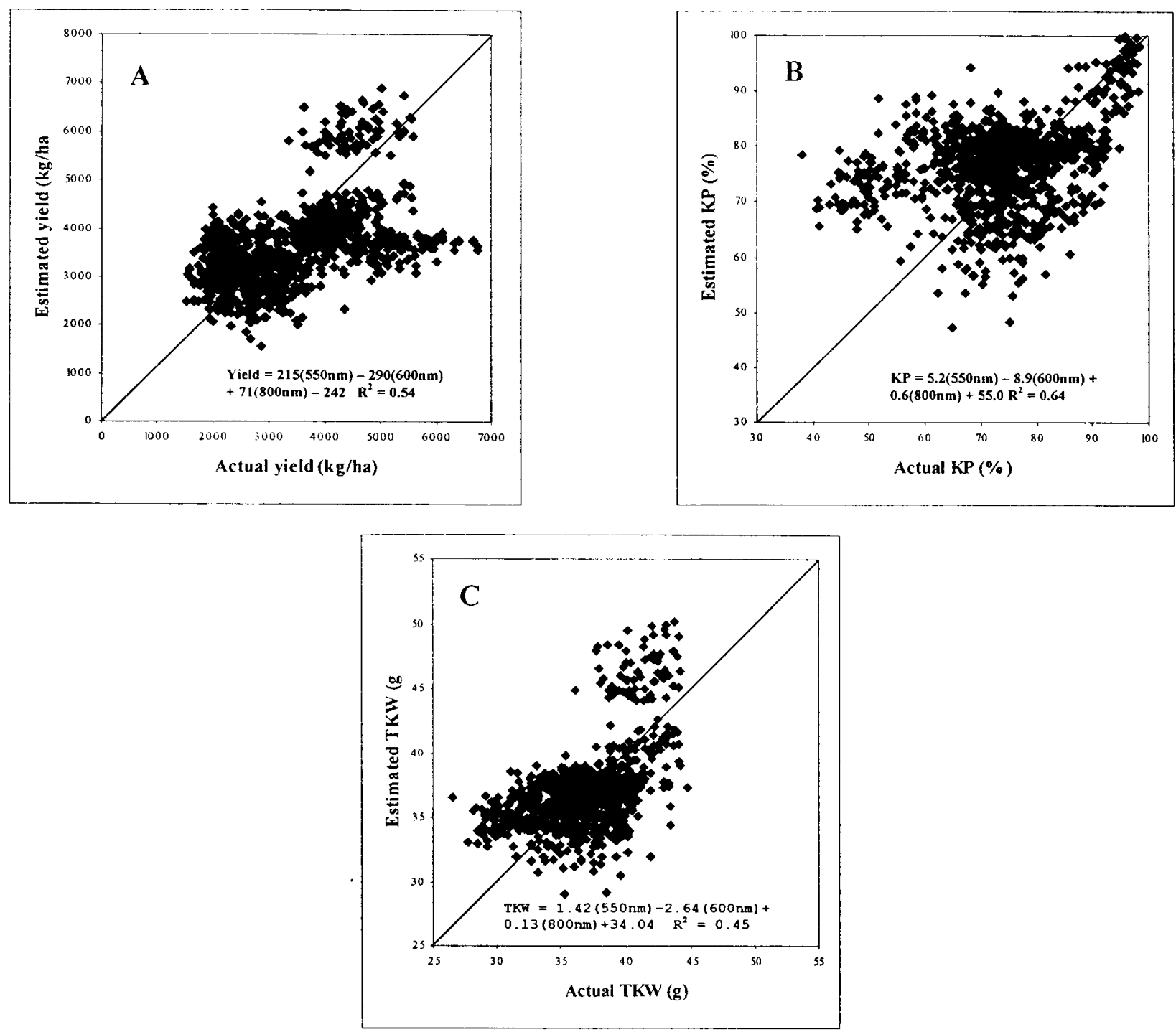

Fig. 2. Relationship between: (A) actual yield and estimated yield; (B) actual kernel plumpness (KP) and estimated KP; and (C) actual thousand kernel weight (TKW) and estimated TKW across the flag (Zadoks 47) and milk (Zadoks 73) growth stages for the barley cultivars Azure and Larker.

Langdon location, the tillering stage appeared to be useful for predicting KP and TKW for all genotypes. Regression models using the 550- and 800-nm wavelengths with either 750 - or $850-\mathrm{nm}$ wavelengths explained 80 to $91 \%$ of the variance in KP and 70 to $83 \%$ of the variance in TKW. Prediction of KP and TKW for all genotypes at GS83 in both locations also was highly significant. At Fargo, the 550-, 800-, and 850-nm wavelengths were useful in estimating $\mathrm{KP}\left(R^{2}=0.68\right.$ to 0.87$)$ and TKW $\left(R^{2}=0.67\right.$ to 0.87$)$, while the 500-, 800-, and 850-nm wavelengths were useful in estimating $\mathrm{KP}\left(R^{2}=0.74\right.$ to 0.93$)$ and TKW $\left(R^{2}=0.67\right.$ to 0.82 ) at Langdon.

The development of canopy reflectance models to predict $\mathrm{KP}$ at the tillering stage was surprising since previous studies found kernel size to be largely determined after the flowering stage (about GS 49 in barley) is complete (Thorne
1966). However, Rasmusson and Cannell (1970) found that yield components of barley are determined at various stages of crop development, depending on environmental influences. The radiometer would also be expected to detect significant soil background effects at the tillering stage with concomitant impacts on the relevance of using canopy reflectance in prediction models. When soil background reflectance is a factor, models using visible wavelengths should be better since reflectance in the red (650-700 nm) and NIR region are more influenced at low vegetation cover (Tucker 1979; Ahlrichs and Bauer 1983; Elliott and Regan 1993). The planting density was preset at a moderately high rate (mean stand counts of ca. 200 plants $\mathrm{m}^{-2}$ ) to minimize soil effects on canopy reflectance, but the Langdon location did have slightly higher (7\%) stand establishment compared to the Fargo location. This may explain why the regression 
models to predict KP and TKW at Fargo had a combination of three visible wavelengths, while models at the Langdon location had combinations of 550-nm and NIR wavelengths at the early growth stages. However, with the likelihood of soil background reflectance influences at early growth stages, the highly significant models that were found at GS83 in both locations for KP and TKW, and the previous studies on determination of kernel size following anthesis (Thorne 1966), GS83 would be the recommended stage to use for predicting KP and TKW.

\section{Vegetation Index Models}

The relationships between simple ratio indices, NDVI, MCARI, and OSAVI with yield are presented in Tables 4 and 5. In general, vegetation indices were inadequate predictors of yield over the 5-yr study. As was found previously in this study, growth stage and genotype affected the ability to use canopy reflectance in estimating agronomic performance. It appears that the NIR/G, NDVI-Green, and MCARI VIs may be useful at early (GS47) growth stages to predict yield (Table 4). However, the most consistent VIs to predict yield were NIR/R and NDVI-Red at the GS47-GS73 stages. This may be due to the probable strong relation of maximum LAI, yield, and VIs that occur at these growth stages compared to VIs made earlier or later in the growing season (Wiegand et al. 1991). Peñuelas et al. (1997) found that NDVI relationships to yield were best determined in barley at early growth stages (biomass $<1500 \mathrm{~g} \mathrm{~m}^{-2}$ ), because NDVI values reached a saturation level $(0.8)$ and did not increase when biomass exceeded $1500 \mathrm{~g} \mathrm{~m}^{-2}$. Regression models using logarithmic or power functions of NDVI did not significantly improve the $R^{2}$ in this study (data not shown), in contrast to what has been found with other NDVI yield models (Ma et al. 2001). Use of NDVIs or simple ratio indices to predict yield appears to be optimum in barley at early growth stages (up to anthesis, where maximum LAI is attained).

Similar to what was found for yield, VIs also were not good predictors of KP or TKW in this study (data not shown), and this relationship was affected by growth stage and genotype. The relationship between VIs and KP or TKW was best at GS24 and GS47. This was unexpected, but was similar to what was found when using simple threewavelength models. NIR/R and MCARI VIs had the best relationship to $\mathrm{KP}\left(0.33<R^{2}<0.77\right.$ and $0.16<R^{2}<0.73$, respectively) and TKW $\left(0.35<R^{2}<0.58\right.$ and $0.05<R^{2}<$ 0.65 , respectively) in this study. Since KP and TKW could be estimated at early growth stages, soil background effects were probably not influencing reflectance measurements because both NIR/R and MCARI are responsive to background reflectance (Daughtry et al. 2000). As was indicated previously, it was unexpected to find significant (but low $R^{2}$ ) models at early growth stages, since KP and TKW are usually not determined until after anthesis (Thorne 1966).

\section{Application of Models for Predicting Agronomic Performance}

The objective of this research was to develop predictive models using canopy reflectance to estimate the agronomic performance in barley. Genotype and crop growth stage variables had significant effects on developing a model that could be universally applied. Nevertheless, this study did find some tendencies that appear to be in common. The two wavelengths that are very important in assessing agronomic performance variables of yield, KP, and TKW are at $550 \mathrm{~nm}$ (green) and $800 \mathrm{~nm}$ (NIR). This agrees with models to predict sugar beet yield by Clevers (1997). The growth stages that appear to be most consistent in assessing yield, KP, and TKW are near GS73, and perhaps as early as GS24 for KP and TKW. When combining data across GS47 to GS73, the $600-\mathrm{nm}$ (red) band also significantly contributed to the model, particularly for KP and TKW. Our results found a consistent positive association between reflectance in the green (550-nm) and NIR (800 and $850 \mathrm{~nm}$ ) wavelengths and agronomic performance, and a consistent negative association between reflectance at red (600-700-nm) wavelengths and agronomic performance. This agrees with previous studies (Clevers 1997; Thenkabail et al. 2000). Predictive models to estimate yield, KP, and TKW in barley using the $550-\mathrm{nm}, 600-\mathrm{nm}$, and 800-nm wavelengths were generated and actual agronomic performance vs. estimated performance (calculated using regression models) is presented in Fig. 2. These models used only the cultivars Azure and Larker and are more representative of current barley cultivar performance than the genotype ND B112. The models are composed of values from the GS47 to GS73 growth stages across all environments. When these models were tested for individual genotypes at different environments, regression equations were usually not significant except in 1991. Since there was an extreme range of agronomic performance due to wide variation in weather across the years and locations in this study, it would not be surprising to find that the generalized equations in Fig. 2 were not good predictors of performance in years with dry or drought conditions. In addition, the generalized models were not as useful when productivity was high. Different models for predicting yield or other agronomic performance variables under high productivity conditions ( $\left.>5000 \mathrm{~kg} \mathrm{ha}^{-1}\right)$ may be necessary, as has been suggested by Ma et al. (2001) for soybean.

\section{CONCLUSION}

Remote sensing that utilized a multispectral radiometer to measure canopy reflectance was evaluated as a potential tool to estimate agronomic performance (yield, KP, TKW) in barley. The stage of crop development at which measurements are made is critical to development of prediction models, as has been found by others (Clevers 1997). In this study, yield, KP, and TKW were best predicted near GS73, where plant biomass and LAI is near maximum. It also may be possible to predict $\mathrm{KP}$ and TKW at earlier (GS24-GS47) growth stages. The wavelengths that are most useful in predictive models were variable, depending on growth stage and genotype, but the 550-nm and 800 -nm bands were clearly critical for the development of generalized equations for prediction of agronomic performance. While VIs were generally not found to be significant in this study, the NIR/R and NDVI-Red were the most useful in predicting agronomic performance. NIR/G and MCARI also may be useful at early growth stages. 
Barley genotype also was an important factor in developing predictive models, particularly when they are very dissimilar genetically, as was found in this study using Azure, Larker, and ND B112 barley. The barley genotype ND B112 has slightly higher LAI compared to the cultivars Azure and Larker, but yielded significantly lower at most environments (Table 1). While LAI is certainly an important component for determination of yield potential, differences among genotypes exist with respect to their ability to convert light energy to the stored product (yield). Therefore, development of one generalized model to accurately predict yield for all cultivars of a specific crop may not be feasible due to inherent differences among cultivars with respect to agronomic performance under identical environmental conditions. However, in many of the major cereal production areas, there is increasing genetic uniformity among cultivars, which may make it possible to develop a single model that could reasonably predict agronomic performance across a range of host cultivars. Further studies using several similar genotypes that are currently used in barley production and focusing on wavelengths near the 550-nm, 600-nm, and various NIR bands around the GS73 stage would likely result in improved models to predict agronomic performance in barley.

\section{ACKNOWLEDGMENTS}

The authors gratefully acknowledge the technical assistance of Brenda Lippert. A portion of this research was conducted at North Dakota State University by the first two authors.

Aase, J. K., Millard, J. P. and Brown, B. S. 1986. Spectral radiance estimates of leaf area and leaf phytomass of small grains and native vegetation. IEEE Trans. Geos. Rem. Sens. 24: 685-692.

Ahlrichs, J. S. and Bauer, M. E. 1983. Relation of agronomic and multispectral reflectance characteristics of spring wheat canopies. Agron. J. 75: 987-993.

American Society of Brewing Chemists. 1992. Methods of analysis. Am. Soc. Brewing Chem., St. Paul, MN.

Bauer, M. E. 1975. The role of remote sensing in determining the distribution and yield of crops. Adv. Agron. 27: 271-304.

Clevers, J. G. P. W. 1988. The derivation of a simplified reflectance model for the estimation of leaf area index. Remote Sens. Environ. 25: 53-69.

Clevers, J. G. P. W. 1997. A simplified approach for yield prediction of sugar beet based on optical remote sensing data. Remote Sens. Environ. 61: 221-228.

Daughtry, C. S. T., Walthall, C. L., Kim, M. S., Brown de Colstoun, E. and McMurtrey, J. E. III. 2000. Estimating corn leaf chlorophyll concentration from leaf and canopy reflectance. Remote Sens. Environ. 74: 229-239.

Elliott, G. A. and Regan, K. L. 1993. Use of reflectance measurements to estimate early cereal biomass production on sandplain soils. Aust. J. Exp.. Agric. 33: 179-183.

Fetch, T. G., Jr. and Steffenson, B. J. 1994. Identification of Cochliobolus sativus isolates expressing differential virulence on tworow barley genotypes in North Dakota. Can. J. Plant Pathol. 16: 202-206.

Hatfield, J. L. and Pinter, P. J., Jr. 1993. Remote sensing for crop protection. Crop Prot. 12: 403-413.
Huete, A. R. 1988. A soil-adjusted vegetation index (SAVI) Remote Sens. Environ. 25: 295-309.

Knipling, E. B. 1970. Physical and physiological basis for the reflectance of visible and near-infrared radiation from vegetation. Remote Sens. Environ. 1: 155-159.

Lorenzen, B. and Jensen, A. 1991. Spectral properties of a barley canopy in relation to the spectral properties of single leaves and the soil. Remote Sens. Environ. 37: 23-34.

Ma, B. L., Dwyer, L. M., Costa, C., Cober, E. R. and Morrison, M. 2001. Early prediction of soybean yield from canopy reflectance measurements. Agron. J. 93: 1227-1234.

Maas, S. J. 1993. Within-season calibration of modeled wheat growth using remote sensing and field sampling. Agron. J. 85: 669-672.

Pederson, V. D. and Fiechtner, G. M. 1980. A low-cost, compact data acquisition system for recording visible and infrared reflection from barley canopies. Pages $71-75$ in P. S. Teng and S. V. Krupa eds. Crop loss assessment. Proc. of E. C. Stakman Comm. Symp., St. Paul, MN. Minnesota Misc. Publ. 7.

Peñuelas, J., Isla, R. Filella, I. and Araus, J. L. 1997. Visible and near-infrared reflectance assessment of salinity effects on barley. Crop Sci. 37: 198-202.

Rasmusson, D. C. and Cannell, R. Q. 1970. Selection for grain yield and components of yield in barley. Crop Sci. 10: 51-54.

Rondeaux, G., Steven, M. and Baret, F. 1996. Optimization of soiladjusted vegetation indices. Remote Sens. Environ. 55: 95-107. SAS Institute, Inc. 1999. SAS onlineDoc ${ }^{\circledR}$, Version 8. SAS Institute Inc., Cary, NC.

Steel, R. G. and Torrie, J. H. 1980. Principles and procedures of statistics. 2nd ed. McGraw-Hill Book Co., New York, NY. 633 pp. Steffenson, B. J., Hayes, P. M. and Kleinhofs, A. 1996. Genetics of seedling and adult plant resistance to net blotch (Pyrenophora teres f. teres) and spot blotch (Cochliobolus sativus) in barley. Theor. Appl. Genet. 92: 552-558.

Thenkabail, P. S., Smith, R. B. and De Pauw, E. 2000. Hyperspectral vegetation indices and their relationships with agricultural crop characteristics. Remote Sens. Environ. 71: 158-182. Thorne, G. N. 1966. Physiological aspects of grain yield in cereals. Pages 79-97 in F. L. Milthorpe and J. D. Ivins, eds. The growth of cereals and grasses. Butterworths Press, London, UK.

Tucker, C. J. 1979. Red and photographic infrared linear combinations for monitoring vegetation. Remote Sens. Environ. 8: 127-150.

Wiegand, C. L., Maas, S. J., Aase, J. K., Hatfield, J. L., Pinter, P. J., Jr., Jackson, R. D., Kanemasu, E. T. and Lapitan, R. L. 1992. Multisite analysis of spectral-biophysical data for wheat. Remote Sens. Environ. 42: 1-21.

Wiegand, C. L. and Richardson, A. J. 1990. Use of spectral vegetation indices to infer leaf area, evapotranspiration and yield: I. Rationale. Agron. J. 82: 623-629.

Wiegand, C. J., Richardson, A. J., Escobar, D. E. and Gerbermann, A. H. 1991. Vegetation indices in crop assessments. Remote Sens. Environ. 35: 105-119.

Wiegand, C. J., Richardson, A. J. and Kanemasu, E. T. 1979. Leaf area index estimates for wheat from LANDSAT and their implications for evapotranspiration and crop modeling. Agron. J. 71: 336-342.

Zadoks, J. C., Chang, T. T. and Konzak, C. F. 1974. A decimal code for the growth stages of cereals. Weed Res. 14: 415-421. 
This article has been cited by:

1. Nima Ahmadian, Sahar Ghasemi, Jean-Pierre Wigneron, Reinhard Zölitz. 2016. Comprehensive study of the biophysical parameters of agricultural crops based on assessing Landsat 8 OLI and Landsat 7 ETM+ vegetation indices. GIScience \& Remote Sensing 53:3, 337-359. [CrossRef] 